\title{
General decay of solutions to a one-dimensional thermoelastic beam with variable coefficients
}

Baowei Feng ${ }^{1}$ and Haiyan $\mathrm{Li}^{2^{*}}$

\section{"Correspondence:}

lihaiyanmath@163.com

${ }^{2}$ School of Mathematics and

Information Science, North Minzu

University, Yinchuan, 750021, P.R.

China

Full list of author information is

available at the end of the article

\begin{abstract}
The vibrations of flexible structures in practice are described by nonlinear models of strings, beams, plates, and so on. This paper is concerned with longitudinal vibrations of a thermoelastic beam equation. Our main result is the general decay of the system. Using the multiplier method and some properties of the convex functions, we establish the general decay of energy.
\end{abstract}

MSC: 35L35; 35B35; 93D15

Keywords: flexible structure; longitudinal vibrations; decay; memory; convexity

\section{Introduction}

Generally speaking, the vibrations of flexible structures in practice are described by nonlinear models of strings, beams, plates, and so on. The linearized vibrations of flexible structures are usually governed by partial differential equations, in particular, by the second-order wave equation and the fourth-order Euler-Bernoulli beam equation [1]. Up till now, there are many results concerning the stability of wave and plate equations by adding some types of damping, for example, internal damping, boundary damping, thermal damping, and so on, most of which can be found in the literature. For general decay results on the wave equation, here we mention the works by Cao and Yao [2], Guesmia and Messaoudi [3], Said-Houari, Messaoudi, and Guesmia [4], Messaoudi [5-7], Messaoudi and Al-Gharabli [8, 9], Messaoudi and Soufyane [10], Mustafa and Messaoudi [11], Tatar [12], and $\mathrm{Wu}[13]$. When the body vibrates, the balance of linear momentum reads

$$
m u_{t t}-\sigma_{x}=f
$$

where $\sigma$ is the stress. If the body is nonuniform, that is,

$$
\sigma=\sigma\left(u_{x}, u_{x t}\right)=p(x) u_{x}+2 \delta(x) u_{x t}
$$

then we can derive the equation of longitudinal vibrations of a flexible structure (see, e.g., Liu and Liu [14])

$$
m(x) u_{t t}-\left(p(x) u_{x}+2 \delta(x) u_{x t}\right)_{x}=f
$$

(c) The Author(s) 2017. This article is distributed under the terms of the Creative Commons Attribution 4.0 International License (http://creativecommons.org/licenses/by/4.0/), which permits unrestricted use, distribution, and reproduction in any medium, provided you give appropriate credit to the original author(s) and the source, provide a link to the Creative Commons license, and indicate if changes were made. 
where $u(x, t)$ represents the longitudinal displacement of a particle. The functions $m(x)$, $p(x)$, and $\delta(x)$ denote the mass per unit length of the structure, the coefficient of internal material damping, and a positive function related to the stress acting on the body, respectively. For this equation, Gorain [15] established the exponential stability of the solution.

For the thermal effect in flexible structures, that is,

$$
\theta_{t}+q_{x}-\kappa u_{t x}=0
$$

where $q(x, t)$ denotes the heat flux vector, and $\theta(x, t)$ is the temperature difference, we we can find the physical background in Carlson [16]. If we assume that the heat flux satisfies a different thermal law, we can obtain flexible structures with different thermal effects. For example, if the heat flux satisfies

$$
q(t)+(1-\alpha) \theta_{x}(t)+\alpha \int_{0}^{\infty} g(s) \theta_{x}(t-s) d s=0, \quad \alpha \in(0,1),
$$

then we get the flexible structures with the Coleman-Gurtin law. The limit cases $\alpha=0$ and $\alpha=1$ correspond to the Fourier and Gurtin-Pipkin cases. Misra et al. [17] studied the thermoelastic flexible structure with Fourier law:

$$
\left\{\begin{array}{l}
m(x) u_{t t}-\left(p(x) u_{x}+2 \delta(x) u_{x t}\right)_{x}+\kappa \theta_{x}=f \\
\theta_{t}-\theta_{x x}+\kappa u_{x t}=0
\end{array}\right.
$$

and proved the global well-posedness of the system. In addition, they established the exponential decay of energy. It is a remarkable fact that the assumption of Fourier's law causes an unrealistic property that a sudden disturbance at some point will be felt instantly everywhere else in the material. Green and Naghdi developed a damped model, called thermoelasticity of type III (see, e.g., [18-23]),

$$
\left\{\begin{array}{l}
u_{t t}-\alpha u_{x x}++\beta \theta_{x}=f, \\
\theta_{t t}-\delta \theta_{x x}+\gamma u_{x t t}-\kappa \theta_{x t t}=0 .
\end{array}\right.
$$

For decay results of this system, we refer to Quintanilla and Racke [24] and Zhang and Zuazua [25] (see also [26]). For flexible structures with thermal effect, we also mention the work of Alves et al. [27], where the authors studied the thermoelastic flexible structure with second sound and proved the well-posedness and stability of the system.

Taking into account all considerations mentioned, in this paper, we consider the following longitudinal vibrations of a thermoelastic beam equation with past history:

$$
\begin{aligned}
& m(x) u_{t t}-\left(p(x) u_{x}+2 \delta(x) u_{x t}\right)_{x}+\kappa \theta_{x t}=0, \\
& \theta_{t t}-\alpha \theta_{x x}-\int_{0}^{\infty} g(s) \theta_{x x}(t-s) d s+\kappa u_{x t}=0
\end{aligned}
$$

where $x \in[0, L]$, and the constant $\kappa$ is the coupling coefficient. The boundary conditions are given by

$$
u(0, t)=u(L, t)=0, \quad \theta(0, t)=\theta(L, t)=0, \quad t \geq 0,
$$


and the initial conditions are

$$
u(x, 0)=u_{0}(x), \quad u_{t}(x, 0)=u_{1}(x),\left.\quad \theta(x, t)\right|_{t \leq 0}=\theta_{0}(x, t), \quad \theta_{t}(x, 0)=\theta_{1}(x) .(1.4)
$$

Our goal in this paper is to establish a general decay of solutions to problem (1.1)-(1.4) with exponential and polynomial decays as only particular cases. We use the multiplier method and some properties of convex functions to establish a general decay result.

To deal with the memory, motivated by Dafermos [28] and Giorgi et al. [29, 30], we define the new variable $\eta=\eta^{t}(x, s)$ by

$$
\eta^{t}(x, s)=\theta(x, t)-\theta(x, t-s), \quad(t, s) \in[0, \infty) \times \mathbb{R}^{+} .
$$

Then

$$
\eta_{t}+\eta_{s}=\theta_{t}, \quad(x, t, s) \in \mathbb{R}^{n} \times \mathbb{R}^{+} \times \mathbb{R}^{+} .
$$

It follows from (1.5) that

$$
\int_{0}^{\infty} g(s) \theta_{x x}(t-s) d s=\int_{0}^{\infty} g(s) d s \theta_{x x}-\int_{0}^{\infty} g(s) \eta_{x x}^{t}(s) d s
$$

Assuming for simplicity that $\alpha-\int_{0}^{\infty} g(s)=1$, problem (1.1)-(1.4) is transformed into the new problem

$$
\begin{aligned}
& m(x) u_{t t}-\left(p(x) u_{x}+2 \delta(x) u_{x t}\right)_{x}+\kappa \theta_{x t}=0, \\
& \theta_{t t}-\theta_{x x}+\int_{0}^{\infty} g(s) \eta_{x x}(s) d s+\kappa u_{x t}=0, \\
& \eta_{t}^{t}+\eta_{s}^{t}=\theta_{t}, \\
& u(x, 0)=u_{0}(x), \quad u_{t}(x, 0)=u_{1}(x), \quad \eta^{t}(x, 0)=0, \quad x \in[0, L], \\
& \left.\theta(x, t)\right|_{t \leq 0}=\theta_{0}(x, t), \quad \theta_{t}(x, 0)=\theta_{1}(x), \quad x \in[0, L], \\
& \eta^{0}(x, s)=\eta_{0}(x, s), \quad(x, s) \in[0, L] \times \mathbb{R}^{+}, \\
& u(0, t)=u(L, t)=0, \quad \theta(0, t)=\theta(L, t)=0, \quad t \geq 0 .
\end{aligned}
$$

The plan of the paper is as follows. In Section 2, we give some assumptions and our main results. The proof of the general decay result is given in Section 3.

\section{Assumptions and main results}

By $L^{q}(0, L)(1 \leq q \leq \infty)$ and $H^{1}(0, L)$ we denote the standard Lebesgue integral and Sobolev spaces. The norm in a space $B$ is denoted by $\|\cdot\|_{B}$. For simplicity, we use $\|u\|$ instead of $\|u\|_{2}$ when $q=2$.

Now we give some assumptions used in this paper.

(A1) The functions $m(x), p(x), \delta(x):[0, L] \rightarrow \mathbb{R}^{+}$are functions of class $C^{1}(0, L)$, and there exist positive constants $m_{1}, m_{2}, p_{1}, p_{2}, \delta_{1}$, and $\delta_{2}$ such that

$$
m_{1} \leq m(x) \leq m_{2}, \quad p_{1} \leq p(x) \leq p_{2}, \quad \text { and } \quad \delta_{1} \leq \delta(x) \leq \delta_{2} .
$$


(A2) The function $g: \mathbb{R}^{+} \rightarrow \mathbb{R}^{+}$is a $C^{1}$ a nonincreasing function satisfying

$$
g(0)>0, \quad 1-\int_{0}^{\infty} g(s) d s=l>0
$$

and there exists a positive constant $a_{0}<m_{2}$ such that, for any $u \in H_{0}^{1}(0, L)$,

$$
a_{0} \int_{0}^{L} u_{x}^{2} d x \leq \int_{0}^{L} m(x) u_{x}^{2} d x-\int_{0}^{L}\left(\int_{0}^{\infty} g(s) d s\right) u_{x}^{2} d x
$$

In addition, there exists an increasing strictly convex function $G: \mathbb{R}^{+} \rightarrow \mathbb{R}^{+}$of class $C^{1}\left(\mathbb{R}^{+}\right) \cap C^{2}\left(\mathbb{R}^{+}\right)$satisfying

$$
G(0)=G^{\prime}(0)=0, \quad \lim _{t \rightarrow \infty} G^{\prime}(t)=\infty,
$$

and

$$
\int_{0}^{\infty} \frac{g(s)}{G^{-1}\left(-g^{\prime}(s)\right)} d s+\sup _{s \in \mathbb{R}^{+}} \frac{g(s)}{G^{-1}\left(-g^{\prime}(s)\right)}<\infty .
$$

To consider the new variable $\eta$, we define the weighted $L^{2}$-spaces

$$
\mathcal{M}=L_{g}^{2}\left(\mathbb{R}^{+}, H_{0}^{1}(0, L)\right)=\left\{\eta: \mathbb{R}^{+} \rightarrow H_{0}^{1}: \int_{0}^{\infty} g(s)\left\|\eta_{x}(s)\right\|^{2} d s<\infty\right\},
$$

which is a Hilbert space endowed with inner product and norm

$$
(\eta, \zeta)_{\mathcal{M}}=\int_{0}^{\infty} g(s)\left(\eta_{x}(s), \zeta_{x}(s)\right) d s \quad \text { and } \quad\|\eta\|_{\mathcal{M}}^{2}=\int_{0}^{\infty} g(s)\left\|\eta_{x}(s)\right\|^{2} d s
$$

Now we define the phase space

$$
\mathcal{H}=H_{0}^{1}(0, L) \times L^{2}(0, L) \times H_{0}^{1}(0, L) \times L^{2}(0, L) \times \mathcal{M}
$$

equipped with the norm

$$
\|(u, v, \theta, \vartheta, \eta)\|_{\mathcal{H}}^{2}=\left\|u_{x}\right\|^{2}+\|v\|^{2}+\left\|\theta_{x}\right\|+\|\vartheta\|+\|\eta\|_{\mathcal{M}}^{2} .
$$

Using semigroup theory, we can easily prove the existence of solutions to problem (1.6)(1.12); see, for example, Alves et al. [27].

Theorem 2.1 Assume that (2.1)-(2.2) hold. Let $U(t)=\left(u, u_{t}, \theta, \theta_{t}, \eta\right)$. If the initial data $U_{0} \in \mathcal{H}$, then problem (1.6)-(1.12) has a unique mild solution $U(t) \in C([0, \infty), \mathcal{H})$ with $U(0)=U_{0}$.

Define the energy $E(t)$ of problem (1.6)-(1.12) by

$$
E(t)=\frac{1}{2}\left[\int_{0}^{L} m(x) u_{t}^{2} d x+\int_{0}^{L} p(x) u_{x}^{2} d x+\int_{0}^{L} \theta_{t}^{2} d x+\int_{0}^{L} \theta_{x}^{2} d x+\|\eta\|_{\mathcal{M}}^{2}\right] .
$$

The general decay result can be given in the following theorem. 
Theorem 2.2 Assume that (2.1)-(2.5) hold. Let $\left(u_{0}, u_{1}, \theta_{0}(\cdot, 0), \theta_{1}, \eta_{0}\right) \in \mathcal{H}$ begiven. Suppose that there exists a constant $C \geq 0$ such that, for any $s>0$,

$$
\left\|\eta_{0 x}\right\| \leq C
$$

Then there exist positive constants $k_{1}, k_{2}, \epsilon_{0}$ such that, for any $t \in \mathbb{R}^{+}$,

$$
E(t) \leq k_{1} H^{-1}\left(k_{2} t\right)
$$

where

$$
H(s)=\int_{s}^{1} \frac{1}{\tau G^{\prime}\left(\epsilon_{0} \tau\right)} d \tau
$$

\section{General decay}

In this section, to prove Theorem 2.2, we establish a general decay of solutions to problem (1.6)-(1.12). We need the following technical lemmas.

Lemma 3.1 For the energy $E(t)$ defined in (2.6), there exists a constant $c_{1}>0$ such that, for any $t>0$,

$$
E^{\prime}(t) \leq-2 \int_{0}^{L} \delta(x) u_{x t}^{2} d x+\int_{0}^{L} g^{\prime}(s)\left\|\eta_{x}(s)\right\|^{2} d s \leq 0
$$

Proof Multiplying (1.6) by $u_{t}$ and (1.7) by $\theta_{t}$ and then integrating the result over $(0, L)$, we easily get the desired estimate (3.1).

Lemma 3.2 Under the assumptions of Theorem 2.2, for the functional $\phi(t)$ defined by

$$
\phi(t)=\int_{0}^{L} m(x) u(t) u_{t}(t) d x+\kappa \int_{0}^{L} \theta_{x}(t) u(t) d x
$$

there exists a positive constant $c_{1}$ such that, for any $t>0$,

$$
\phi^{\prime}(t) \leq-\frac{1}{2} \int_{0}^{L} p(x) u_{x}^{2} d x+\int_{0}^{L} m(x) u_{x}^{2} d x+\frac{\kappa}{2} \int_{0}^{L} \theta_{x}^{2} d x+c_{1} \int_{0}^{L} u_{x t}^{2} d x,
$$

where $c_{1}=\frac{\kappa}{2 \lambda_{1}}$, and $\lambda_{1}>0$ is the Poincaré constant.

Proof Taking the derivative of $\phi(t)$ with respect to $t$ and using (1.6), we get

$$
\begin{aligned}
\phi^{\prime}(t)= & -2 \int_{0}^{L} \delta(x) u_{x} u_{x t} d x-\int_{0}^{L} p(x) u_{x}^{2} d x+\int_{0}^{L} m(x) u_{t}^{2} d x \\
& +\kappa \int_{0}^{L} \theta_{x} u_{t} d x .
\end{aligned}
$$

Young's inequality, Poincaré's inequality, and (2.1) give us

$$
-2 \int_{0}^{L} \delta(x) u_{x} u_{x t} d x \leq \frac{1}{2} \int_{0}^{L} p(x) u_{x}^{2} d x+\frac{4 \delta_{2}^{2}}{p_{1}} \int_{0}^{L} u_{x t}^{2} d x
$$


and

$$
\kappa \int_{0}^{L} \theta_{x} u_{t} d x \leq \frac{\kappa}{2} \int_{0}^{L} \theta_{x}^{2} d x+\frac{\kappa}{2 \lambda_{1}} \int_{0}^{L} u_{x t}^{2} d x
$$

which, together with (3.3), give us (3.2).

Lemma 3.3 Under the assumptions of Theorem 2.2, the functional $\psi(t)$ defined by

$$
\psi(t)=\int_{0}^{L} \theta(t) \theta_{t}(t) d x+\kappa \int_{0}^{L} u_{x}(t) \theta(t) d x
$$

satisfies

$$
\psi^{\prime}(t) \leq-\frac{1}{2} \int_{0}^{L} \theta_{x}^{2} d x+\left(\frac{\kappa}{2}+1\right) \int_{0}^{L} \theta_{t}^{2} d x+\frac{\kappa}{2 \lambda_{1}} \int_{0}^{L} u_{x t}^{2} d x+\frac{1-l}{2}\|\eta\|_{\mathcal{M}}^{2}
$$

for all $t>0$.

Proof It follows from (1.7) that

$$
\psi^{\prime}(t)=-\int_{0}^{L} \theta_{x}^{2} d x+\int_{0}^{L} \theta_{t}^{2} d x-\int_{0}^{L} \theta_{x}(t) \int_{0}^{\infty} g(s) \eta_{x}(s) d s+\kappa \int_{0}^{L} u_{x} \theta_{t} d x
$$

Using Young's and Poincaré's inequalities, we infer that

$$
-\int_{0}^{L} \theta_{x}(t) \int_{0}^{\infty} g(s) \eta_{x}(s) d s d x \leq \frac{1}{2} \int_{0}^{L} \theta_{x}^{2} d x+\frac{1-l}{2}\|\eta\|^{2}
$$

and

$$
\kappa \int_{0}^{L} u_{x} \theta_{t} d x \leq \frac{\kappa}{2} \int_{0}^{L} \theta_{t}^{2} d x+\frac{\kappa}{2 \lambda_{1}} \int_{0}^{L} u_{x t}^{2} d x
$$

which, along with (3.5), implies (3.4).

Lemma 3.4 Under the assumptions of Theorem 2.2, for the functional $\chi(t)$ defined by

$$
\chi(t)=-\int_{0}^{L} \theta_{t}(t) \int_{0}^{\infty} g(s) \eta(s) d s d x
$$

there exists a positive constant $c_{2}$ such that, for any $t>0$,

$$
\begin{aligned}
\chi^{\prime}(t) \leq & -\frac{1-l}{2} \int_{0}^{L} \theta_{t}^{2} d x+\frac{\kappa^{2}}{2 \lambda_{1}} \int_{0}^{L} u_{x t}^{2} d x+2(1-l)\|\eta\|_{\mathcal{M}}^{2} \\
& -c_{2} \int_{0}^{\infty} g^{\prime}(s)\left\|\eta_{x}(s)\right\|^{2} d s .
\end{aligned}
$$


Proof Direct differentiation using (1.7) implies

$$
\begin{aligned}
\chi^{\prime}(t)= & \underbrace{\int_{0}^{L} \theta_{x}\left(\int_{0}^{\infty} g(s) \eta_{x}(s) d s\right) d x}_{:=I_{1}} \underbrace{\int_{0}^{L}\left(\int_{0}^{\infty} g(s) \eta_{x}(s) d s\right)^{2} d x}_{:=I_{2}} \\
& \underbrace{-\kappa \int_{0}^{L} u_{t} \int_{0}^{\infty} g(s) \eta_{x}(s) d s d x}_{:=I_{4}} \underbrace{-\int_{0}}_{-\int_{0}^{L} \theta_{t} \int_{0}^{\infty} g(s) \eta_{t}(s) d s d x} .
\end{aligned}
$$

It follows from Hölder's and Young's inequalities that

$$
I_{1} \leq \frac{1}{2} \int_{0}^{L} \theta_{x}^{2} d x+\frac{1-l}{2}\|\eta\|_{\mathcal{M}}^{2}, \quad I_{2} \leq(1-l)\|\eta\|_{\mathcal{M}}^{2}
$$

and

$$
I_{3} \leq \frac{\kappa^{2}}{2 \lambda_{1}} \int_{0}^{L} u_{x t}^{2} d x+\frac{1-l}{2}\|\eta\|_{\mathcal{M}}^{2}
$$

Noting (1.8) and using Young's inequality, we have

$$
\begin{aligned}
I_{4} & =-(1-l) \int_{0}^{L} \theta_{t}^{2} d x-\int_{0}^{L} \theta_{t} \int_{0}^{\infty} g^{\prime}(s) \eta(s) d s d x \\
& \leq-\frac{(1-l)}{2} \int_{0}^{L} \theta_{t}^{2} d x-\frac{l_{1}}{2(1-l) \lambda_{1}} \int_{0}^{\infty} g^{\prime}(s)\left\|\eta_{x}(s)\right\|^{2} d s
\end{aligned}
$$

where $l_{1}=-\int_{0}^{\infty} g^{\prime}(s) d s$. Inserting these estimates into (3.7), we get (3.6) with

$$
c_{2}=\frac{l_{1}}{2(1-l) \lambda_{1}} \text {. }
$$

The proof is completed.

We further define the Lyapunov functional $\mathcal{L}(t)$ by

$$
\mathcal{L}(t)=N E(t)+N_{1} \phi(t)+N_{2} \psi(t)+N_{3} \chi(t)
$$

where $N, N_{1}, N_{2}, N_{3}$ are positive constants to be chosen later. First, it is easy to verify that there exists two positive constants $\beta_{1}$ and $\beta_{2}$ such that

$$
\beta_{1} E(t) \leq \mathcal{L}(t) \leq \beta_{2} E(t)
$$

Lemma 3.5 For suitable constants $N, N_{1}, N_{2}, N_{3}>0$, there exist two positive constants $c_{4}$ and $c_{5}$ such that

$$
\mathcal{L}^{\prime}(t) \leq-c_{4} E(t)+c_{5} \int_{0}^{\infty} g(s)\left\|\eta_{x}(s)\right\|^{2} d s
$$


Proof Combining (3.1)-(3.2), (3.4), and (3.6) and using (2.1), we can obtain that, for any $t>0$,

$$
\begin{aligned}
\mathcal{L}^{\prime}(t)= & N E^{\prime}(t)+N_{1} \phi^{\prime}(t)+N_{2} \psi^{\prime}(t)+N_{3} \chi^{\prime}(t) \\
\leq & -\left[2 N \delta_{1}-\left(\frac{m_{2}}{\lambda_{1}}+c_{1}\right) N_{1}-\frac{\kappa}{2 \lambda_{1}} N_{2}-\frac{\kappa^{2}}{2 \lambda_{1}} N_{3}\right] \int_{0}^{L} u_{x t}^{2} d x \\
& -\frac{N_{1} p_{1}}{2} \int_{0}^{L} u_{x}^{2} d x-\left(\frac{N_{2}}{2}-\frac{\kappa}{2} N_{1}\right) \int_{0}^{L} \theta_{x}^{2} d x+c_{3}\|\eta\|_{\mathcal{M}}^{2} \\
& -\left[\frac{1-l}{2} N_{3}-\left(\frac{\kappa}{2}+1\right) N_{2}\right] \int_{0}^{L} \theta_{t}^{2} d x+\left(N-c_{2} N_{3}\right) \int_{0}^{\infty} g^{\prime}(s)\left\|\eta_{x}(s)\right\|^{2} d s,
\end{aligned}
$$

where $c_{3}=N_{1} \frac{1-l}{2}+2 N_{3}(1-l)$.

At this point, we first choose $N_{1}, N_{2}, N_{3}>0$ that satisfy

$$
N_{3}>\frac{\kappa+2}{2(1-l)} N_{2}, \quad N_{2}>\kappa N_{1},
$$

which implies

$$
\frac{N_{2}}{2}-\frac{\kappa}{2} N_{1}>0, \quad \frac{1-l}{2} N_{3}-\left(\frac{\kappa}{2}+1\right) N_{2}>0 .
$$

Then we get that there exist constants $\gamma>0$ and $\mu>0$ such that

$$
\begin{aligned}
\mathcal{L}^{\prime}(t) \leq & -\left[2 N \delta_{1}-\left(\frac{m_{2}}{\lambda_{1}}+c_{1}\right) N_{1}-\frac{\kappa}{2 \lambda_{1}} N_{2}-\frac{\kappa^{2}}{2 \lambda_{1}} N_{3}-\frac{\mu}{\lambda_{1}}\right] \int_{0}^{L} u_{x t}^{2} d x \\
& -\gamma E(t)+c_{3}\|\eta\|_{\mathcal{M}}^{2}+\left(N-c_{2} N_{3}\right) \int_{0}^{\infty} g^{\prime}(s)\left\|\eta_{x}(s)\right\|^{2} d s .
\end{aligned}
$$

Finally, for fixed $N_{1}, N_{2}, N_{3}>0$, we take $N>0$ large enough so that

$$
2 N \delta_{1}-\left(\frac{m_{2}}{\lambda_{1}}+c_{1}\right) N_{1}-\frac{\kappa}{2 \lambda_{1}} N_{2}-\frac{\kappa^{2}}{2 \lambda_{1}} N_{3}-\frac{\mu}{\lambda_{1}}>0, \quad N-c_{2} N_{3}>0,
$$

which, together with (3.10), gives us (3.9).

Lemma 3.6 Under the assumptions of Theorem 2.2, there exists a positive constant $\gamma_{1}>0$ such that, for any $\epsilon_{0}>0$,

$$
G^{\prime}\left(\epsilon_{0} E(t)\right) \int_{0}^{\infty} g(s)\left\|\eta_{x}(s)\right\|^{2} d s \leq-\gamma_{1} E^{\prime}(t)+\gamma_{1} \epsilon_{0} E(t) G^{\prime}\left(\epsilon_{0} E(t)\right) .
$$

Proof We prove this lemma by using the method developed by Guesmia [31]. It follows from (2.3), (2.7), and (3.1) that

$$
\begin{aligned}
\int_{0}^{L} \eta_{x}^{2} d x & \leq 2 \int_{0}^{L}\left(u_{x}^{2}(x, t)+u_{x}^{2}(x, t-s)\right) d x \\
& \leq 4 \sup _{t \geq 0} \int_{0}^{L} u_{x}^{2}(t) d x+2 \sup _{s>0} \int_{0}^{L} u_{0 x}^{2}(x, s) d x
\end{aligned}
$$




$$
\begin{aligned}
& \leq \frac{8}{a_{0}} E(0)+4 \sup _{s>0} \int_{0}^{L}\left(\left(\eta_{x}^{0}\right)^{2}(x, s)+u_{0 x}^{2}(x, 0)\right) d x \\
& \leq \frac{16}{a_{0}} E(0)+4 C:=C_{0},
\end{aligned}
$$

where $C_{0}$ is a positive constant depending on $E(0), a_{0}$, and $C$.

First, if $g^{\prime}\left(s_{0}\right)=0$ for some $s_{0} \geq 0$, then from (2.5) we infer that $g\left(s_{0}\right)=0$. Since $g(t)$ is nonincreasing and nonnegative, we know that $g(s)=0$ for any $s \geq s_{0}$. Therefore

$$
\int_{0}^{\infty} g(s) \eta_{x}^{2}(s) d s=\int_{0}^{s_{0}} g(s) \eta_{x}^{2}(s) d s
$$

Without loss of generality, we can assume that $g^{\prime}(s)<0$ for $s \in \mathbb{R}^{+}$.

For $s \in \mathbb{R}^{+}$, we define $K(s)=\frac{s}{G^{-1}(s)}$. By the properties of $G$ we know that $K(0)=G^{\prime}(0)=0$, and hence the function $K(s)$ is nondecreasing. Taking into account (3.12), we obtain that, for any $s_{1}>0$,

$$
K\left(-s_{1} g^{\prime}(s)\left\|\eta_{x}\right\|^{2}\right) \leq K\left(-C_{0} s_{1} g^{\prime}(s)\right)
$$

Therefore, for $\epsilon_{0}, \tau_{1}>0$,

$$
\begin{aligned}
& \int_{0}^{L} g(s)\left\|\eta_{x}(s)\right\|^{2} d s \\
& \quad=\frac{1}{\tau_{1} G^{\prime}\left(\epsilon_{0} E(t)\right)} \int_{0}^{\infty} G^{-1}\left(-s_{1} g(s)\left\|\eta_{x}\right\|^{2}\right) \frac{\tau_{1} G^{\prime}\left(\epsilon_{0} E(t)\right) g(s)}{-s_{1} g(s)} K\left(-s_{1} g(s)\left\|\eta_{x}\right\|^{2}\right) d s \\
& \quad \leq \frac{1}{\tau_{1} G^{\prime}\left(\epsilon_{0} E(t)\right)} \int_{0}^{\infty} G^{-1}\left(-s_{1} g(s)\left\|\eta_{x}\right\|^{2}\right) \frac{\tau_{1} G^{\prime}\left(\epsilon_{0} E(t)\right) g(s)}{-s_{1} g(s)} K\left(-C_{0} s_{1} g^{\prime}(s)\right) d s \\
& \quad \leq \frac{1}{\tau_{1} G^{\prime}\left(\epsilon_{0} E(t)\right)} \int_{0}^{\infty} G^{-1}\left(-s_{1} g(s)\left\|\eta_{x}\right\|^{2}\right) \frac{C_{0} \tau_{1} G^{\prime}\left(\epsilon_{0} E(t)\right) g(s)}{G^{-1}\left(-C_{0} s_{1} g^{\prime}(s)\right)} d s .
\end{aligned}
$$

Now we denote the conjugate function of the convex function $G$ by $G^{*}$ (see, e.g., Arnold [32], Daoulatli et al. [33], Lasiecka and Doundykov [34], and Lasiecka and Tataru [35]), that is,

$$
G^{*}(s)=\sup _{t \in \mathbb{R}^{+}}(s t-G(t)) .
$$

Then,

$$
G^{*}(s)=s\left(G^{\prime}\right)^{-1}(s)-G\left[\left(G^{\prime}\right)^{-1}(s)\right]
$$

for $s \geq 0$ is the Legendre transform of $G$, which satisfies

$$
t_{1} t_{2} \leq G\left(t_{1}\right)+G^{*}\left(t_{2}\right) \text {. }
$$

for $t_{1}, t_{2} \geq 0$. Denoting

$$
t_{1}=G^{-1}\left(-s_{1} g(s)\left\|\eta_{x}\right\|^{2}\right), \quad t_{2}=\frac{C_{0} \tau_{1} G^{\prime}\left(\epsilon_{0} E(t)\right) g(s)}{G^{-1}\left(-C_{0} s_{1} g^{\prime}(s)\right)}
$$


from (3.13) we see that

$$
\begin{aligned}
\int_{0}^{\infty} g(s)\left\|\eta_{x}(s)\right\|^{2} d s \leq & \frac{-s_{1}}{\tau_{1} G^{\prime}\left(\epsilon_{0} E(t)\right)} \int_{0}^{\infty} g^{\prime}(s)\left\|\eta_{x}(s)\right\|^{2} d s \\
& +\frac{1}{\tau_{1} G^{\prime}\left(\epsilon_{0} E(t)\right)} \int_{0}^{\infty} G^{*}\left(\frac{C_{0} \tau_{1} G^{\prime}\left(\epsilon_{0} E(t)\right) g(s)}{G^{-1}\left(-C_{0} s_{1} g^{\prime}(s)\right)}\right) d s
\end{aligned}
$$

which, together with (3.1) and the inequality $G^{*}(s) \leq s\left(G^{\prime}\right)^{-1}(s)$, gives

$$
\begin{aligned}
\int_{0}^{\infty} & g(s)\left\|\eta_{x}(s)\right\|^{2} d s \\
\leq & \frac{-2 s_{1}}{\tau_{1} G^{\prime}\left(\epsilon_{0} E(t)\right)} E^{\prime}(t) \\
& \quad+C_{0} \int_{0}^{\infty} \frac{g(s)}{G^{-1}\left(-C_{0} s_{1} g^{\prime}(s)\right)}\left(G^{\prime}\right)^{-1}\left(\frac{C_{0} \tau_{1} G^{\prime}\left(\epsilon_{0} E(t)\right) g(s)}{G^{-1}\left(-C_{0} s_{1} g^{\prime}(s)\right)}\right) d s .
\end{aligned}
$$

Using (2.5), we denote

$$
\sup _{s>0} \frac{g(s)}{G^{-1}\left(-g^{\prime}(s)\right)}=b<+\infty .
$$

By the properties of $G$ we get the function $\left(G^{\prime}\right)^{-1}$ is nondecreasing. Then taking $s_{1}=\frac{1}{C_{0}}$, we conclude from (3.14) that

$$
\begin{aligned}
\int_{0}^{\infty} g(s)\left\|\eta_{x}(s)\right\|^{2} d s \leq & \frac{-2}{C_{0} \tau_{1} G^{\prime}\left(\epsilon_{0} E(t)\right)} E^{\prime}(t) \\
& +C_{0}\left(G^{\prime}\right)^{-1}\left(C_{0} b \tau_{1} G^{\prime}\left(\epsilon_{0} E(t)\right)\right) \int_{0}^{\infty} \frac{g(s)}{G^{-1}\left(-g^{\prime}(s)\right)} d s .
\end{aligned}
$$

Similarly, denoting

$$
\int_{0}^{\infty} \frac{g(s)}{G^{-1}\left(-g^{\prime}(s)\right)} d s=d<+\infty
$$

and picking $\tau_{1}=\frac{1}{C_{0} b}$, we infer from (3.15) that

$$
\int_{0}^{\infty} g(s)\left\|\eta_{x}(s)\right\|^{2} d s \leq \frac{-2 b}{G^{\prime}\left(\epsilon_{0} E(t)\right)} E^{\prime}(t)+C_{0} d \epsilon_{0} E(t)
$$

which gives (3.11).

Proof of Theorem 2.2 Multiplying (3.9) by $G^{\prime}\left(\epsilon_{0} E(t)\right)$ and using (3.11), we obtain

$$
G^{\prime}\left(\epsilon_{0} E(t)\right) \mathcal{L}^{\prime}(t) \leq-\left(c_{4}-c_{5} \gamma_{1} \epsilon_{0}\right) E(t) G^{\prime}\left(\epsilon_{0} E(t)\right)-\gamma_{1} c_{5} E^{\prime}(t)
$$

Taking $\epsilon_{0}$ small enough so that $c_{4}-c_{5} \gamma_{1} \epsilon_{0}>0$, we get that there exists a constant $c_{6}>0$ such that

$$
G^{\prime}\left(\epsilon_{0} E(t)\right) \mathcal{L}^{\prime}(t)+\gamma_{1} c_{5} E^{\prime}(t) \leq-c_{6} E(t) G^{\prime}\left(\epsilon_{0} E(t)\right) .
$$


Define

$$
\mathcal{E}(t)=\tau\left(G^{\prime}\left(\epsilon_{0} E(t)\right) \mathcal{L}(t)+\gamma_{1} c_{5} E(t)\right)
$$

with $\tau>0$. Noting that $G^{\prime}\left(\epsilon_{0} E(t)\right)$ is nonincreasing, we can easily get from (3.8) that

$$
\mathcal{E}^{\prime}(t) \leq-c_{6} \tau G^{\prime}\left(\epsilon_{0} E(t)\right) E(t)
$$

Using (3.8) and the inequality $G^{\prime}\left(\epsilon_{0} E(t)\right) \leq G^{\prime}\left(\epsilon_{0} E(0)\right)$, we can get that there exist two positive constants $\beta_{3}$ and $\beta_{4}$ such that

$$
\beta_{3} E(t) \leq \mathcal{E}(t) \leq \beta_{4} E(t)
$$

Choose $\tau>0$ small enough so that

$$
\mathcal{E}(t) \leq E(t), \quad \mathcal{E}(0) \leq 1
$$

Noting that $s \mapsto s G^{\prime}\left(\epsilon_{0} s\right)$ is nondecreasing, from (3.17) we obtain

$$
\mathcal{E}^{\prime}(t) \leq-c_{7} \mathcal{E}(t) G^{\prime}\left(\epsilon_{0} \mathcal{E}(t)\right)
$$

with $c_{7}=c_{6} \tau$. This shows that $(H(\mathcal{E}(t)))^{\prime} \geq c_{7}$, where

$$
H(t)=\int_{t}^{1} \frac{1}{s G^{\prime}\left(\epsilon_{0} s\right)} d s
$$

Integrating it over $[0, t]$, we see that

$$
H(\mathcal{E}(t)) \geq c_{7} t+H(\mathcal{E}(0))
$$

which, together with (3.18) and $H(1)=0$, implies

$$
H(\mathcal{E}(t)) \geq c_{7} t .
$$

Since $H^{-1}$ is decreasing, we get

$$
\mathcal{E}(t) \leq H^{-1}\left(c_{7} t\right)
$$

Then (2.8) follows from the equivalence $\mathcal{E}(t) \sim E(t)$.

Remark 3.7 Condition (2.5) allows $g$ to have a decay rate close to $\frac{1}{t}$, and the rate of energy decay (2.8) depends on $g$.

Finally, we give three examples to illustrate several rates of energy decay, which can be found in Guesmia [31]. 
Example 1 Let $g(t)=\frac{\mu}{(1+t)^{p}}$ with $p>1$ and $\mu>0$ small enough so that (2.3) holds. Condition (2.5) is satisfied for $G(t)=t^{1+\frac{1}{q}}$ with $q \in\left(0, \frac{p-1}{2}\right)$. Then from (2.8) we get that there exists a constant $\beta_{5}>0$ such that, for any $q \in\left(0, \frac{p-1}{2}\right)$,

$$
E(t) \leq \frac{\beta_{5}}{(1+t)^{q}}
$$

Example 2 Let $g(t)=\mu e^{-(\ln (2+t))^{p}}$ with $p>1$ and $\mu>0$ small enough so that (2.3) holds. For

$$
G(t)=\int_{0}^{t}(-\ln s)^{1-\frac{1}{q}} e^{-(-\ln s)^{\frac{1}{q}}} d s,
$$

when $t$ is near zero, (2.5) holds with with $q \in(1, p)$. Then from (2.8) we get that there exist two constants $\beta_{5}>0$ and $\beta_{6}>0$ such that, for any $q \in(1, p)$,

$$
E(t) \leq \beta_{5} e^{-\beta_{6}(\ln (1+t))^{q}} .
$$

Example 3 Let $g(t)=\mu e^{-(1+t)^{p}}$ with $p \in(0,1)$ and $\mu>0$ small enough so that $(2.3)$ holds. For

$$
G(t)=\int_{0}^{t}(-\ln s)^{1-\frac{1}{q}} d s
$$

when $t$ is near zero, (2.5) holds with $q \in\left(1, \frac{p}{2}\right)$. Then from (2.8) we get that there exist two constants $\beta_{5}>0$ and $\beta_{6}>0$ such that, for any $q \in\left(1, \frac{p}{2}\right)$,

$$
E(t) \leq \beta_{5} e^{-\beta_{6} t^{q}}
$$

\section{Conclusions}

In this work, we consider a model to the longitudinal vibrations of a beam equation with thermoviscoelastic damping. Motivated by Dafermos [28], we introduce a new variable, and the system is transformed into a new system. We give the global existence of solutions without proof. The main result is the energy decay of solutions. Under suitable assumptions, we established a general decay result of energy for the initial value problem by using the energy perturbation method and some properties of convex functions. Finally, we give three examples, which can be found in Guesmia [31], to illustrate several rates of energy decay.

Acknowledgements

The authors would like to thank the referees for careful reading this paper and for valuable suggestions to improve the paper.

Funding

Baowei Feng was supported by the NSFC (No. 11701465) and the Fundamental Research Funds for the Central

Universities (No. JBK170127). Haiyan Li was supported by the Scientific Research Funds for the Ningxia Universities (No. NGY2017162). 
Authors' contributions

Both authors contributed equally and significantly in writing this paper. Both authors read and approved the final manuscript.

\section{Author details}

1 Department of Economic Mathematics, Southwestern University of Finance and Economics, Chengdu, 611130, P.R. China. ${ }^{2}$ School of Mathematics and Information Science, North Minzu University, Yinchuan, 750021, P.R. China.

\section{Publisher's Note}

Springer Nature remains neutral with regard to jurisdictional claims in published maps and institutional affiliations.

Received: 10 August 2017 Accepted: 23 October 2017 Published online: 02 November 2017

\section{References}

1. Nandi, PK, Gorain, GC, Kar, S: A note on stability of longitudinal vibrations of an inhomogeneous beam. Appl. Math. 3 , 19-23 (2012)

2. Cao, X, Yao, P: General decay rate estimates for viscoelastic wave equation with variable coefficients. J. Syst. Sci. Complex. 27, 836-852 (2014)

3. Guesmia, A, Messaoudi, SA: A general decay result for a viscoelastic equation in the presence of past and finite history memories. Nonlinear Anal., Real World Appl. 13, 476-485 (2012)

4. Said-Houari, B, Messaoudi, SA, Guesmia, A: General decay of solutions of a nonlinear system of viscoelastic wave equations. Nonlinear Differ. Equ. Appl. 18, 659-684 (2011)

5. Messaoudi, SA: General decay of solutions of a weak viscoelastic equation. Arab. J. Sci. Eng. 36, 1569-1579 (2011)

6. Messaoudi, SA: General decay of solutions of a viscoelastic equation. J. Math. Anal. Appl. 341, 1457-1467 (2008)

7. Messaoudi, SA: General decay of solution energy in a viscoelastic equation with a nonlinear source. Nonlinear Anal. 69, 2589-2598 (2008)

8. Messaoudi, SA, Al-Gharabli, MM: A general decay result of a nonlinear system of wave equations with infinite memories. Appl. Math. Comput. 259, 540-551 (2015)

9. Messaoudi, SA, Al-Gharabli, MM: A general decay result of a viscoelastic equation with past history and boundary feedback. Z. Angew. Math. Phys. 66, 1519-1528 (2015)

10. Messaoudi, SA, Soufyane, A: General decay of solutions of a wave equation with a boundary control of memory type. Nonlinear Anal., Real World Appl. 11, 2896-2904 (2010)

11. Mustafa, MI, Messaoudi, SA: General stability result for viscoelastic wave equations. J. Math. Phys. 53, 053702 (2012)

12. Tatar, NE: Arbitrary decays in linear viscoelasticity. J. Math. Phys. 52, 013502 (2011)

13. Wu, ST: General decay of solutions for a nonlinear system of viscoelastic wave equations with degenerate damping and source terms. J. Math. Anal. Appl. 406, 34-48 (2013)

14. Liu, K, Liu, Z: Exponential decay of energy of the Euler-Bernoulli beam with locally distributed Kelvin-Voigt damping SIAM J. Control Optim. 36, 1086-1098 (1998)

15. Gorain, GC: Exponential stabilization of longitudinal vibrations of an inhomogeneous beam. J. Math. Sci. 198, 245-251 (2014)

16. Carlson, DE: Linear thermoelasticity. In: Handbook of Physics (1972)

17. Misra, S, Alves, M, Gorain, GC, Vera, O: Stability of the vibrations of an inhomogeneous flexible structure with thermal effect. Int. J. Dyn. Control 3, 354-362 (2015)

18. Green, AE, Naghdi, PM: A re-examination of the basic postulates of thermodynamics. Proc. R. Soc. Lond. Ser. A 432 171-194 (1991)

19. Green, AE, Naghdi, PM: On undamped heat waves in an elastic solid. J. Therm. Stresses 15, 253-264 (1992)

20. Green, AE, Naghdi, PM: Thermoelasticity without energy dissipation. J. Elast. 31, 189-208 (1993)

21. Green, AE, Naghdi, PM: A unified procedure for construction of theories of deformable media, I. Classical continuum physics. Proc. R. Soc. Lond. Ser. A 448, 335-356 (1995)

22. Green, AE, Naghdi, PM: A unified procedure for construction of theories of deformable media, II. Generalized continua. Proc. R. Soc. Lond. Ser. A 448, 357-377 (1995)

23. Green, AE, Naghdi, PM: A unified procedure for construction of theories of deformable media, III. Mixtures of interacting continua. Proc. R. Soc. Lond. Ser. A 448, 379-388 (1995)

24. Quintanilla, R, Racke, R: Stability in thermoelasticity of type III. Discrete Contin. Dyn. Syst., Ser. B 3, 383-400 (2003)

25. Zhang, X, Zuazua, E: Decay of solutions of the system of thermoelasticity of type III. Commun. Contemp. Math. 5, 1-59 (2003)

26. Fatori, LH, Rivera, JEM: Energy decay for hyperbolic thermoelasticity systems of memory type. Q. Appl. Math. 59 , 441-458 (2001)

27. Alves, MS, Gamboa, P, Gorain, GC, Rambaud, A, Vera, O: Asymptotic behavior of a flexible structure with Cattaneo type of thermal effect. Indag. Math. 27, 821-834 (2016)

28. Dafermos, CM: Asymptotic stability in viscoelasticity. Arch. Ration. Mech. Anal. 37, 297-308 (1970)

29. Giorgi, C, Grasseli, M, Pata, V: Well-posedness and longtime behavior of the phase-field model with memory in a history space setting. Q. Appl. Math. 59, 701-736 (2001)

30. Giorgi, C, Marzocchi, A, Pata, V: Asymptotic behavior of a semilinear problem in heat conduction with memory. Nonlinear Differ. Equ. Appl. 5, 333-354 (1998)

31. Guesmia, A: Asymptotic stability of abstract dissipative systems with infinite memory. J. Math. Anal. Appl. 382 $748-760(2011)$

32. Arnold, Vl: Mathematical Methods of Classical Mechanics. Springer, New York (1989)

33. Daoulatli, M, Lasiecka, I, Toundykov, D: Uniform energy decay for a wave equation with partially supported nonlinear boundary dissipation without growth restrictions. Discrete Contin. Dyn. Syst. 2, 67-95 (2009)

34. Lasiecka, I, Doundykov, D: Energy decay rates for the semilinear wave equation with nonlinear localized damping and a nonlinear source. Nonlinear Anal. 64, 1757-1797 (2006)

35. Lasiecka, I, Tataru, D: Uniform boundary stabilization of semilinear wave equations with nonlinear boundary damping. Differ. Integral Equ. 6, 507-533 (1993) 